\title{
Italian validation of INQoL, a quality of life questionnaire for adults with muscle diseases
}

\author{
V. A. Sansone ${ }^{a}$, M. Panzeri ${ }^{a}$, M. Montanari ${ }^{b}$, G. Apolone ${ }^{b}$, S. Gandossini ${ }^{a}$, M. R. Rose ${ }^{c}$,

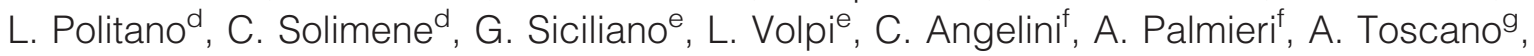 \\ O. Musumeci' , T. Mongini ${ }^{\text {h }}$ L. Vercellih, R. Massa', M. B. Panico', M. Grandi and G. Meola ${ }^{a}$ \\ ${ }^{a}$ Department Neurology, University of Milan, IRCCS Policlinico San Donato; ${ }^{b}$ Mario Negri Institute, Laboratory for Translational and \\ Outcome Research, Department Oncology, Milan, Italy; ' Department Neurology, Kings College, University of London, London, UK; \\ ${ }^{\mathrm{d}}$ Department of Cardiomyology and Clinical Genetic, University of Naples, Naples; ${ }^{\mathrm{e}}$ Department of Neurosciences, University of Pisa, Pisa; \\ ${ }^{\mathrm{f}}$ Department of Neurosciences, University of Padua, Padua; ${ }^{\mathrm{g}}$ Neurological and Neurosurgery Institute, University of Messina, Messina; \\ ${ }^{\mathrm{h}}$ Department of Neurosciences, University of Turin, Turin, ${ }^{\mathrm{i}}$ Department of Neurosciences, University of Tor Vergata and Fond IRCCS S \\ Lucia, Rome; and ${ }^{\mathrm{j}}$ Respiratory Physiopathology, Costa Masnaga, Como, Italy
}

\section{Keywords:}

INQoL, muscle diseases, quality of life, SF-36

Received 14 November 2009 Accepted 20 January 2010
Background and purpose: A quality of life (QoL) questionnaire for neuromuscular diseases was recently constructed and validated in the United Kingdom in a sample of adult patients with a variety of muscle disorders. Preliminary results suggested it could be a more relevant and practical measure of QoL in muscle diseases than generic health measures of QoL. The purpose of our work was: (i) To validate INQoL in Italy on a larger sample of adult patients with muscle diseases (ii) to compare INQoL to SF-36.

Methods: We have translated into Italian and applied language adaptations to the original UK INQoL version. We studied 1092 patients with different muscle disorders and performed (i) test-retest reliability $(n=80)$; (ii) psychometric $(n=345)$, knowngroup $(n=1092)$, external criterion $(n=70)$, and concurrent validity with SF-36 $(n=183)$.

Results: We have translated and formally validated the Italian version of INQoL confirming and extending results obtained in the United Kingdom. In addition to good results in terms of reliability, known-group and criterion validity, a comparison with the SF-36 scales showed a stronger association between INQoL total index and SF-36 physical $(r=-0.72)$ than mental $(r=-0.38)$ summary health indexes. When considering comparable domains of INQoL and SF-36 with respect to an objective measure of muscle strength assessment (MMRC), regression analysis showed a stronger correlation using INQoL rather than SF-36 scores.

Conclusions: INQoL is recommended to assess QoL in muscle diseases because of its ability to capture physical limitations that are specifically relevant to the muscle condition.

\section{Introduction}

Muscle diseases are heterogeneous conditions that share the property of physical disability usually attributed to progressive muscle weakness. Generic healthrelated quality of life questionnaires, including the widely used SF-36, have not been specifically con-

Correspondence: V. A. Sansone, Department of Neurology, IRCCS Policlinico San Donato, Via Morandi, 30, 20097 - San Donato Milanese (Mi), Milan, Italy (tel.: + 3902 52774556;

fax: + 3902 5274717; e-mail: valeria.sansone@unimi.it). structed and validated for patients with muscle diseases [1-9]. Whilst patients with muscle disorders may share some functional limitations with other patients with chronic diseases, some issues considered in generic health measures (e.g., ability to walk long distances, climbing stairs) may be superfluous to those with muscle disorders (who are may be in a wheel-chair) and some issues that are particularly relevant (e.g., muscle stiffness, independence) may be omitted. Therefore, a muscle disease-specific QoL measure may be more relevant and consequently more sensitive for patients with muscle disease. The Individualized Neuromuscular 
QoL (INQoL) is a muscle disease-specific QoL measure developed in the United Kingdom [10]. Its theoretical basis, development, content and construct validity, reliability, and some limited responsiveness data suggest that INQoL has the potential to be a useful assessment of QoL in patients with muscle disease. Because QoL may be an outcome for international trials and studies of muscle disease, there is an advantage in having INQoL available in other languages including Italian. Therefore, we decided to validate INQoL for use in Italy. This process entails a formal translation of the INQoL but also a cultural validation obtained by submitting the INQoL questionnaire to a large group of Italian patients with an assessment of its reliability and psychometric properties. We also took this opportunity to formally compare the performance of INQoL alongside that of the SF-36 Health Survey.

\section{Methods}

During the entire process of translation and validation, a total of 1092 subjects were recruited in eight Italian Muscle Clinics from the five diagnostic categories (Table S1) through a multistep recruiting approach. Among these patients, 80 patients were recruited for the test-retest reliability evaluation, 345 for the psychometric evaluation, 70 for the criterion validity, 1092 for the known-group validity, and 183 completed both INQOL and SF-36 questionnaires for the concurrent validation. Recruitment depended on the availability of the data from the different Centers involved in the study, and on the willingness of patients to complete all questionnaires and testing. There was no a priori choice of the patients and inclusion in one evaluation or another.

\section{Recruitment and data collection}

Subjects had to be older than 18 years. On the basis of history data and laboratory data, patients were excluded if they had major comorbidities unrelated to muscle disease such as arthritis, respiratory disease (other than neuromuscular respiratory weakness), cardiovascular disease (other than muscle disease-related cardiomyopathy), and cognitive impairment defined as Mini Mental State Examination (MMSE) < 22 [11]. Only patients fitting specific diagnostic criteria for each disease group were included in the study (Table S1).

Data on age, gender, education, and disease duration were collected. All assessments were performed during the course of the patients' usual out-patient visit to their muscle clinic. The questionnaires were administered in three phases. In phase 1, subject just completed INQoL. For phase 2, a proportion of the phase 1 subjects
( $n=80$ ) returned to the clinic 3 weeks later to complete INQoL again so as to obtain data on test-retest reliability. A 3-week period was considered to be short enough to minimize the likelihood that change would occur in any of the dimensions but long enough to reduce the chance the patients would recall their previous answers. In phase 3 , a new cohort of patients $(n=183)$ were asked to complete both INQoL and SF-36. The patients were recruited for each phase consecutively by attendance at the Muscle Clinics at the eight participating centers with no a priori choice of the patients.

Data from medical records and from the INQoL were collected in paper form, and a data manager from each center inserted clinical reports, ratings, and data from each patient (anonymously recorded with letter and number coding) in an electronic database. The clinical research forms, paper questionnaires, and the electronic formats from each center were sent to the coordinator center. The insertion of data and coding were checked by two different people at the coordinator center site (from paper to electronic database) to increase precision.

Muscle testing was performed in the clinic by a muscle disease specialist according to a standardized manual muscle strength testing protocol. A total of 15 muscles were tested bilaterally (shoulder abductors and adductors, elbow flexors and extensors, wrist flexors and extensors, sternocleidomastoid muscles, thumb opponents, long finger flexors, hip flexors, knee flexors and extensors, ankle dorsiflexors, plantar flexors, and extensor digit brevis). The five-point modified Medical Research Council (MRC) scale was used allowing calculation of an MRC sum score ranging from 0 to 150 $[12,13]$.

Cognitive status was assessed by MMSE scores corrected for age and education [11].

The Italian Version of SF-36 was used [4].

The study had ethical approval at the Coordinator's site (IRCCS PSD) and locally at each of the site's Ethical Committees. All subjects signed consent to the study.

\section{INQoL questionnaire}

INQoL consists of 45 questions within 10 sections (Table S2). Four of these refer to the impact of common muscle disease symptoms [weakness, myotonia (locking), pain, and fatigue]. Five look at the degree and importance of impact of the muscle disease on particular areas of life (activities, independence, relationships, emotions, and body image). The last section looks at treatment and its effects and expectations.

Participants respond using a seven-point Likert scale giving their view of the degree of impact of a symptom 
or the degree of impact of muscle disease on an aspect of their life together with the importance that they attach to each item thus allowing a patient weighted score to be given for each section. The final score from each section is presented as a percentage of the maximum detrimental impact with a higher percentage indicating greater symptom impact or worse QoL. A composite score can also be obtained from five preselected sections (scales) assessing the impact of the muscle disease on particular areas of life, this representing overall QoL. Participant's perception of treatment is represented by two scores, reflecting the trade-off between the positive and negative effects of current treatment and expectations for the future. In conclusion, INQOL includes 45 items, structured in 10 sections, yielding 11 scores and one total score.

\section{INQOL translation and linguistic adaptation}

We added space at the end of the questionnaire for the patients to write comments on the questionnaire or on their muscle disease. These free text comments were assigned a coded ID number allowing anonymous analysis. A thematic analysis of the comments was made, separating themes already considered in the questionnaire from new themes mentioned by the patients. This analysis allowed us to make considerations on cultural similarities and differences between the Italian and UK populations and, if necessary, to apply changes to the final Italian version.

INQoL was translated using a simplified approach based on published guidelines [14]. INQoL was translated into Italian by an official English-Italian translator and then back translated from Italian into English by another English-Italian translator. Assessment of conceptual and linguistic equivalence was made during the translation process from English into Italian and vice versa and after test-retest reliability. Any linguistic changes in the wording of the final Italian version were discussed with the UK center and between the Italian centers with the support of the official English-Italian translator. INQoL was usually self-completed in 5-10 min, but a physician was available for explanation of the questions or for any other clarification when requested. If help was required, the physician recorded on a separate note what were the reasons for helping the patients (unclear wording, visual, motor, or cognitive impairment).

\section{Statistical analysis plan}

We applied criteria outlined by the Scientific Advisory Committee of the Medical Outcome Trust for the
Italian validation of INQoL [14,15]. After appropriate translation, language and cultural adaptations, we considered: (i) reliability, including internal consistency and reproducibility and (ii) validity, including psychometric evaluation (scaling and grouping success), construct-related (known-group validity) and criterionrelated validity (INQoL's correlation with objective and functional measures of muscles strength). In addition, we compared INQoL to SF-36 (concurrent validity) to: (i) study the direction and strength of the association between INQOL and SF-36, (ii) see whether INQoL could better capture the physical limitations because of the muscle condition than the more generic SF-36.

\section{Reliability}

Three-week test-retest reliability was assessed using several methods: correlation coefficients, ICC [16], Bland and Altman's method [17]; limits of agreement are calculated to assess the size of differences between the first and second administrations of the questionnaire on the same patient. Internal consistency reliability using Cronbach's alpha [18] was also determined.

\section{Validity}

Psychometric evaluation Psychometric evaluation was assessed using multitrait scaling, including item-item, item-scale and scale-scale correlations. In particular, item internal consistency (correlation between an item in that domain and the domain score computed from all other items in that domain) and item discriminant validity (correlation between an item of a domain and the other domains) were evaluated. A satisfactory item-scale correlation was set when $r \geq 0.40$ implying appropriate inclusion of items within a specific domain.

Known-group (correlation between INQOL and variables describing cognitive impairment, demographic and clinical parameters) and Criterion-related (correlation with objective measures of muscle strength) validity Construct and criterion validity of the iNQOL was assessed in relation to external criteria known to have an expected impact on questionnaires scores such as age, gender, type of diagnosis, and of an objective measure of muscle strength. Analysis of variance was used to calculate the $F$ statistic, defined as the ratio between-group and with-group (error) variance, for each scale and external (or criterion) group.

Concurrent validity: comparison between INQoL index and SF-36 We used Spearman's correlation coefficient to estimate the association between INQoL and SF-36 scales and summary indexes. 
First, the association between the INQOL scales and total score with each of the scales and two SF-36 summary indexes were estimated to assess the nature (direction and strength) of the association between INQOL and an external well-known generic questionnaire. Then, to test whether the new specific questionnaire was different (better or worse) in measuring specific health concepts relevant for the disease under evaluation, selected scales of both questionnaires (INQOL pain versus SF-36 bodily pain; INQOL weakness versus Sf-36 vitality; INQOL fatigue versus SF-36 vitality; INQOL activity versus SF-36 physical functioning; and INQOL emotion versus SF-36 mental health) were compared in terms of association (correlation) with a standardized objective measure of muscle strength (MegaMRC).

\section{Results}

Table 1 shows the characteristics of each of the subsamples used for each specific analysis, for a total of 1092 patients included in five subsamples assembled during the development and validation process to be specifically tested according to specific research topics: reliability $($ No. $=80)$, psychometric evaluation (345), known-group validity (1092), criterion validity (70), and comparison with the Sf-36 (183).

\section{Reliability}

In the 80 subjects who completed two administrations of INQoL, the difference in mean values between initial and second measurements ranged from 0.99 (locking,

Table 1 Sample characteristics

\begin{tabular}{|c|c|c|c|c|c|}
\hline & $\begin{array}{l}\text { Test-retest } \\
(n=80)\end{array}$ & $\begin{array}{l}\text { Psychometric } \\
\text { evaluation }(n=345)\end{array}$ & $\begin{array}{l}\text { Criterion validity } \\
(n=70)\end{array}$ & $\begin{array}{l}\text { Known-group } \\
\text { validity }(n=1092)\end{array}$ & $\begin{array}{l}\text { SF-36 comparison } \\
(n=183)\end{array}$ \\
\hline Age (years); sex $(\mathrm{M} / \mathrm{F}, \%)$ & $43.9(12.0) ; 65 / 35$ & 42.8 (13.9); 66/34 & 45.9 (13.2); $73 / 27$ & 43.9 (14.9); $59 / 41$ & $44.1(15.3) ; 54 / 46$ \\
\hline Disease duration (years) & $16.2(12.3)$ & $12.9(9.9)$ & $15.7(10.6)$ & $16.3(12.5)$ & $16.0(13.7)$ \\
\hline Education (years) & $10.7(3.9)$ & $10.2(3.4)$ & $10.5(3.2)$ & $10.4(3.7)$ & $11.0(3.5)$ \\
\hline \multicolumn{6}{|l|}{ Diagnosis (\%) } \\
\hline DM & 40.0 & 40.5 & 100 & 38.6 & 39.5 \\
\hline FSHD & 24.3 & 24.8 & - & 23.3 & 18.5 \\
\hline LGMD & 10.0 & 14.5 & - & 17.9 & 19.1 \\
\hline BMD & 18.6 & 13.8 & - & 13.6 & 7.6 \\
\hline IM & 7.1 & 6.4 & - & 6.6 & 15.3 \\
\hline \multicolumn{6}{|l|}{ INQoL domains } \\
\hline Weakness & $46.3(27.7)$ & $47.6(28.9)$ & $36.7(28.1)$ & $47.6(31.9)$ & $46.0(28.8)$ \\
\hline Locking & $27.6(30.3)$ & $27.5(30.6)$ & $32.0(31.0)$ & $27.5(30.7)$ & $28.6(29.7)$ \\
\hline Pain & $24.7(28.9)$ & $25.3(30.5)$ & $12.4(22.5)$ & $24.4(30.0)$ & $25.3(30.3)$ \\
\hline Fatigue & $41.8(29.5)$ & $43.0(30.9)$ & $35.4(29.6)$ & $44.8(30.7)$ & $41.8(30.8)$ \\
\hline Activity & $41.9(29.7)$ & $40.9(29.1)$ & $29.3(27.8)$ & $42.1(29.2)$ & $39.6(27.1)$ \\
\hline Independence & $34.6(32.6)$ & $35.1(31.6)$ & $23.5(29.0)$ & $34.5(32.5)$ & $29.8(30.9)$ \\
\hline Relationships & $18.5(22.0)$ & $17.5(22.3)$ & $13.3(20.3)$ & $16.7(21.1)$ & $13.7(17.8)$ \\
\hline Emotions & $26.2(24.6)$ & $26.5(24.2)$ & $19.6(20.4)$ & $28.4(24.4)$ & $27.1(22.5)$ \\
\hline Body image & $36.7(29.6)$ & $36.0(30.4)$ & $29.3(30.5)$ & $36.0(30.2)$ & $34.0(29.8)$ \\
\hline INQoL index & $33.7(22.3)$ & $33.3(22.8)$ & $8.7(22.3)$ & $33.7(23.1)$ & $31.3(21.0)$ \\
\hline \multicolumn{6}{|l|}{ SF-36 domains } \\
\hline Bodily pain & - & - & - & - & $68.2(30.1)$ \\
\hline Role physical & - & - & - & - & $59.7(41.9)$ \\
\hline Physical functioning & - & - & - & - & $54.1(32.7)$ \\
\hline Vitality & - & - & - & - & $50.9(21.8)$ \\
\hline General health & - & - & - & - & $48.5(23.7)$ \\
\hline Social functioning & - & - & - & - & $69.8(25.8)$ \\
\hline Role emotional & - & - & - & - & $68.6(40.5)$ \\
\hline Mental health & - & - & - & - & $64.8(19.7)$ \\
\hline Mental health Index & - & - & - & - & $40.1(12.5)$ \\
\hline Physical health Index & - & - & - & - & $47.2(10.8)$ \\
\hline \multicolumn{6}{|l|}{ Other scales } \\
\hline MMRC & - & - & $135.6(14.2)$ & - & - \\
\hline MMSE & - & - & $27.3(2.6)$ & - & - \\
\hline
\end{tabular}

DM, myotonic dystrophies; FSHD, facioscapulo humeral dystrophy; LGMD, limb-girdle muscular dystrophies; BMD, Becker muscular dystrophy; IM, inflammatory myopathies; Age, disease duration, education, INQoL domains, SF-36 domains, MMRC, and MMSE values are expressed as means. Standard deviation is in brackets. $n=$ number of patients considered for that procedure/analysis. 
Table 2 Test-retest results

\begin{tabular}{|c|c|c|c|c|c|c|c|}
\hline \multirow[b]{2}{*}{ Domains } & \multirow[b]{2}{*}{ No. of items } & \multirow{2}{*}{$\begin{array}{l}\text { Correlation } \\
\text { coefficient }\end{array}$} & \multirow{2}{*}{$\begin{array}{l}95 \% \text { Confidence } \\
\text { interval } \\
\text { Min-Max }\end{array}$} & \multirow[b]{2}{*}{ ICC } & \multirow{2}{*}{$\begin{array}{l}\text { Mean difference } \\
\text { (SD) }\end{array}$} & \multicolumn{2}{|c|}{ Cronbach's alpha } \\
\hline & & & & & & Test & Retest \\
\hline Muscle weakness & 3 & 0.7272 & $0.7158-0.7382$ & 0.7283 & $-4.47(20.50)$ & 0.8817 & 0.9123 \\
\hline Locking & 3 & 0.7512 & $0.7401-0.7619$ & 0.7538 & 0.99 (20.97) & 0.9002 & 0.9200 \\
\hline Muscle pain & 3 & 0.8184 & $0.8100-0.8265$ & 0.8204 & $0.99(17.20)$ & 0.8981 & 0.9235 \\
\hline Fatigue & 3 & 0.8340 & $0.8263-0.8414$ & 0.8358 & $-1.84(17.25)$ & 0.8779 & 0.9093 \\
\hline Activities & 5 & 0.7722 & $0.7636-0.7804$ & 0.7709 & $-7.34(18.67)$ & 0.8790 & 0.9101 \\
\hline Independence & 3 & 0.7056 & $0.6941-0.7167$ & 0.7056 & $-6.25(23.12)$ & 0.8846 & 0.9165 \\
\hline Relationships & 10 & 0.8513 & $0.8448-0.8575$ & 0.8523 & $-2.73(11.31)$ & 0.8847 & 0.9200 \\
\hline Emotions & 6 & 0.6988 & $0.6859-0.7112$ & 0.7013 & $-2.30(19.38)$ & 0.8853 & 0.9150 \\
\hline Body image & 3 & 0.6930 & $0.6803-0.7053$ & 0.6944 & $-4.41(22.62)$ & 0.8857 & 0.9124 \\
\hline Treatment & 6 & 0.4575 & $0.4377-0.4768$ & 0.4601 & $3.75(30.03)$ & 0.9298 & 0.9455 \\
\hline
\end{tabular}

muscle pain) to -7.34 (activities), with a median of 0 (score range from -100 to 100 ).

Correlation coefficients ranged from 0.83 (fatigue) to 0.69 (body image), with a median value of 0.72 . Even for those domains (activities and independence) where differences between initial and repeat administrations showed higher dispersion of mean values (activities: -7.3; independence: -6.3 ), correlation coefficients were high (0.8 and 0.7 , respectively). Also, the interclass correlation coefficient (ICC) showed a range of consistent values except for the Treatment domain See Table 2 .

Cronbach's alpha was estimated twice in the testretest sample. In both cases, its values were high, varying from 0.88 to 0.95 .

\section{Validity}

Table 3 shows the descriptive statistics of the INQOL questionnaire in terms of mean, standard deviation, and range. The full range of values was actually present.

Table 3 Distribution of INQoL scales and INQoL total index

\begin{tabular}{llll}
\hline INQoL domains & $\begin{array}{l}\text { Mean } \\
(\mathrm{SD})\end{array}$ & $\begin{array}{l}\text { Standard } \\
\text { deviation }\end{array}$ & Range \\
\hline Weakness & 47.6 & 31.9 & $0-100$ \\
Locking & 27.5 & 30.7 & $0-100$ \\
Pain & 24.4 & 30.0 & $0-100$ \\
Fatigue & 44.8 & 30.7 & $0-100$ \\
Activity & 42.1 & 29.2 & $0-100$ \\
Independence & 34.5 & 32.5 & $0-100$ \\
Relationships & 16.7 & 21.1 & $0-100$ \\
Emotions & 28.4 & 24.4 & $0-100$ \\
Body image & 36.0 & 30.2 & $0-100$ \\
Treatment effect & 17.8 & 29.5 & -67 to 100 \\
Treatment expectation & 16.0 & 29.2 & -92 to 100 \\
INQoL index & 33.7 & 23.1 & $0-100$ \\
\hline
\end{tabular}

See text for details.
Estimates according to age and gender are reported later.

\section{Psychometric evaluation}

Table 4 summarizes the results of scaling and grouping tests of INQoL questionnaire.

Cronbach's estimates, evaluating the internal consistency of the scores, always satisfied the levels for group comparison $(>0.70)$. Two scales (relationship and body image) had values $>0.90$, the level requested for individual comparison. In terms of grouping and scaling tests, we observed $100 \%$ success for internal convergence and low frequency of discriminant failures, activity, and independence scores being those with more failures $(8-10 \%)$.

Table 5 shows the univariate correlation between the INQOL Total score and each of the five scales used to assemble it. All scales had a correlation coefficient higher than 0.70 , being the Activity scale the one with the strongest correlation 0.85 . We also tested the multivariable association between the INQOL Total score and each of the five preselected scales used to assemble it to evaluate the nature of the association of each scale with the summary score after adjusting for the reciprocal confounding effect, as well as the amount of variance explained. The five scales explained about the $94 \%$ of variance, and again the Activity scale emerged as the most important factor of the total score.

\section{Construct and criterion validity}

As reported in the Methods section, we tested the association between INQOL scores and selected external variables known to capture the disease characteristics and complexity.

Correlation between INQoL index and clinical parameters Table 6 summarizes the results of the association between INQOL scores and selected variables, 
Table 4 Summary results of tests of item internal consistency and discriminant validity: results from INQoL questionnaire $(n=1092)$

\begin{tabular}{llllll}
\hline Domain & $\begin{array}{l}\text { Cronbach's } \\
\text { alpha }^{\text {a }}\end{array}$ & $\begin{array}{l}\text { Item internal } \\
\text { consistency } \\
\text { range }\end{array}$ & $\begin{array}{l}\text { \% Internal } \\
\text { convergence }^{\mathrm{b}}\end{array}$ & $\begin{array}{l}\text { Discriminant } \\
\text { validity range }^{\mathrm{d}}\end{array}$ & $\begin{array}{l}\text { \% Item discriminant } \\
\text { validity failure }^{\mathrm{e}}\end{array}$ \\
\hline Activity & 0.88 & $0.69-0.76$ & 100.0 & $0.45-0.75$ & 10.0 \\
Independence & 0.90 & $0.77-0.83$ & 100.0 & $0.48-0.81$ & 8.3 \\
Relationship & 0.93 & $0.66-0.80$ & 100.0 & $0.38-0.66$ & 0.0 \\
Emotions & 0.90 & $0.64-0.81$ & 100.0 & $0.32-0.69$ & 4.2 \\
Body image & 0.91 & $0.78-0.86$ & 100.0 & $0.45-0.65$ & 0.0 \\
\hline
\end{tabular}

${ }^{\mathrm{a}}$ Internal consistency reliability; ${ }^{\mathrm{b}}$ correlations between items and hypothesized scale corrected for overlap; ${ }^{\mathrm{c}}$ percentage of the internal correlation coefficients $>0.4$; ${ }^{\mathrm{d}}$ correlations between items of each scale with other scales; ${ }^{\mathrm{e}}$ percentage of correlations of items with own scales lower than correlations with other scales.

Table 5 Correlation matrix between INQoL scales and total INQoL index

\begin{tabular}{|c|c|c|c|c|c|c|}
\hline & QoL index & Activity & Independence & Relationships & Emotions & Body image \\
\hline QoL index & - & 0.86 & 0.81 & 0.73 & 0.81 & 0.82 \\
\hline Activity & 0.86 & - & 0.79 & 0.58 & 0.61 & 0.69 \\
\hline Independence & 0.81 & 0.79 & - & 0.55 & 0.52 & 0.62 \\
\hline Relationships & 0.73 & 0.58 & 0.55 & - & 0.64 & 0.52 \\
\hline Emotions & 0.81 & 0.61 & 0.52 & 0.64 & - & 0.64 \\
\hline Body image & 0.82 & 0.69 & 0.62 & 0.52 & 0.64 & - \\
\hline
\end{tabular}

See text for details.

such as gender, schooling, diagnosis, and disease duration used to test the questionnaire known-group validity. Based on clinical ground and on previous research in this field [19-23], it was hypothesized that (i) scores would be higher in women, (ii) there would be a positive association between quality of life and education (years of schooling) and a negative correlation between quality of life perception and disease duration, (iii) a difference across type of diseases, with lower scores in myotonic dystrophies, because of the known cognitive-behavioral impairment in these disorders. Table 6 confirms our hypothesis regarding gender and disease duration and, to a minor degree, regarding education.

As expected, INQoL total index was significantly lower in myotonic dystrophies compared to INQoL total index in FSHD, LGMD, and inflammatory myopathies $(P<0.0001)$.

As to the association between INQOL Total score and MMSE, we found a negative correlation: for each increase in MMSE score by 1 unit, there was a decrease of INQoL index of 1.5 units $(r=-0.3317$; $P=0.0636$ ).

Correlation between INQoL index and muscle strength $\mathrm{A}$ negative correlation between INQoL index and MegaMRC was observed where the stronger the patient the better is QoL perceived $(r=-0.549 ; P<0.0001)$ : in practical terms, for each increase in MRC score by
1 unit, there is a decrease of INQoL index of 0.958 units. A positive correlation is seen between INQoL index and MIRS. In this case, the more impaired from a functional point of view, the worst is QoL perceived $(r=0.4695 ; P<0.0001)$.

When exploring stratification by MegaMRC scores (cutoff 140/150, data not-shown) and by disease duration (cutoff 10 years of disease), we observed a relationship between these parameters and INQoL: the higher the MegaMRC score, the better the scores for each of the five domains constituting INQoL index (from nine in 'relationship' to one in 'independence'); the longer the disease duration, the worst the scores in each of the five domain constituting INQoL index (from a 3-points worsening in 'emotion' to a 13-points worsening in 'independence').

\section{Concurrent validity}

Correlation between INQoL and SF-36 Table 7 shows the correlation between preselected INQOL and SF-36 scores. When considering the correlation between single comparable domains included in the INQoL and SF-36 questionnaires, on average, the correlation between relevant domains was in the expected negative direction, and most of the time substantial. Of note, the association between the pain scores in each scale $(r=-0.79)$, the INQOL activity and SF-36 physical functioning scores $(r=0.71)$, INQOL weakness and 
Table 6 Summary results of known-group and criterion validity

\begin{tabular}{|c|c|c|c|c|c|}
\hline Gender & $\begin{array}{l}\text { Female }(n=448) \\
\text { Mean }(\mathrm{SD})\end{array}$ & $\begin{array}{l}\text { Male }(n=644) \\
\text { Mean }(\mathrm{SD})\end{array}$ & $P$-value & & \\
\hline \multicolumn{6}{|l|}{ INQoL domains } \\
\hline Weakness & $51.0(28.9)$ & $44.4(29.4)$ & 0.0003 & & \\
\hline Locking & $29.2(31.6)$ & $25.5(29.4)$ & 0.0526 & & \\
\hline Pain & $30.2(31.6)$ & $19.8(27.7)$ & $<0.0001$ & & \\
\hline Fatigue & $50.3(30.0)$ & $40.3(30.5)$ & $<0.0001$ & & \\
\hline Activity & $46(30.4)$ & $39.0(27.9)$ & 0.0001 & & \\
\hline Independence & $40.5(34.2)$ & $30.2(30.7)$ & $<0.0001$ & & \\
\hline Relationships & $18.7(22.7)$ & $14.8(19.8)$ & 0.0034 & & \\
\hline Emotions & $33.8(26.0)$ & $24.2(22.4)$ & $<0.0001$ & & \\
\hline Body image & $40.1(30.4)$ & $32.6(29.6)$ & $<0.0001$ & & \\
\hline INQoL index & $37.8(23.8)$ & $30.3(21.8)$ & $<0.0001$ & & \\
\hline \multirow[t]{2}{*}{ Education (years) } & $1-5(n=555)$ & $6-10(n=247)$ & $11-13(n=218)$ & $>14(n=72)$ & \\
\hline & Mean (SD) & Mean (SD) & Mean (SD) & Mean (SD) & $P$-value \\
\hline \multicolumn{6}{|l|}{ INQoL domains } \\
\hline Weakness & $49.9(29.2)$ & $46.0(29.7)$ & $41.9(28.3)$ & $46.8(30.1)$ & 0.0793 \\
\hline Locking & $27.8(32.0)$ & $29.5(30.7)$ & $25.6(27.1)$ & $25.3(30.4)$ & 0.2178 \\
\hline Pain & $25.9(31.0)$ & $26.4(30.5)$ & $19.2(26.7)$ & $21.4(29.2)$ & 0.0005 \\
\hline Fatigue & $47.8(30.9)$ & $45.1(30.4)$ & $38.6(29.9)$ & $39.4(31.3)$ & 0.0061 \\
\hline Activity & $44.8(30.1)$ & $43.2(29.0)$ & $34.9(26.5)$ & $38.2(27.2)$ & $<0.0001$ \\
\hline Independence & $36.6(33.3)$ & $35.8(32.7)$ & $28.1(29.6)$ & $31.7(31.5)$ & 0.0015 \\
\hline Relationships & 18 (22.6) & $15.9(20.1)$ & $14.6(18.8)$ & $14.9(17.6)$ & 0.0126 \\
\hline Emotions & $31.1(25.9)$ & $27.2(23.8)$ & $22.4(19.9)$ & $29.0(23.4)$ & 0.0001 \\
\hline Body image & $38.1(30.4)$ & $34.0(30.2)$ & $32.6(28.8)$ & $35.3(31.3)$ & 0.0515 \\
\hline INQoL index & $35.9(24.2)$ & $33.4(22.4)$ & $28.5(20.2)$ & $32.3(21.7)$ & 0.0003 \\
\hline \multirow[t]{2}{*}{ Disease duration (years) } & $1-5(n=207)$ & $6-10(n=233)$ & $11-20(n=328)$ & $>21(n=324)$ & \\
\hline & Mean (SD) & Mean (SD) & Mean (SD) & Mean (SD) & $P$-value \\
\hline \multicolumn{6}{|l|}{ INQoL domains } \\
\hline Weakness & $38.3(31.2)$ & $41.5(28.9)$ & $45.7(28.3)$ & $58.0(24.4)$ & $<0.0001$ \\
\hline Locking & $24.9(31.3)$ & $27.2(30.0)$ & $27.1(30.1)$ & $30.1(31.5)$ & 0.4123 \\
\hline Pain & $26.2(31.1)$ & $24.1(28.0)$ & $22.0(30.3)$ & $26.0(30.1)$ & 0.4405 \\
\hline Fatigue & $40.4(32.9)$ & $39.9(31.1)$ & $45.3(30.4)$ & $51.7(27.6)$ & 0.0002 \\
\hline Activity & $34.2(29.4)$ & $36.9(28.0)$ & $41.2(28.2)$ & $53.1(27.1)$ & $<0.0001$ \\
\hline Independence & $23.4(28.9)$ & $26.9(29.5)$ & $34.2(32.0)$ & $49.9(32.2)$ & $<0.0001$ \\
\hline Relationships & $12.6(20.4)$ & $15.3(20.9)$ & $16.4(20.0)$ & $20.6(22.0)$ & 0.0019 \\
\hline Emotions & $24.3(25.0)$ & $25.2(23.5)$ & $26.1(22.4)$ & $32.5(25.0)$ & 0.0017 \\
\hline Body image & $26.8(29.2)$ & $30.0(28.9)$ & $35.4(28.9)$ & $46.1(29.6)$ & $<0.0001$ \\
\hline INQoL index & $27.0(23.4)$ & $29.2(21.4)$ & $32.7(21.2)$ & $41.8(22.3)$ & 0.0096 \\
\hline
\end{tabular}

the SF-36 physical functioning scores $(r=0.72)$. A somewhat lower unsatisfactory association was observed between the INQOL relationships and the SF-36 social functioning scores $(r=-0.45)$. As to the association between relevant summary scores, we observed a stronger, negative association between INQoL index and Physical Health index $(r=-0.7238 ; P<0.0001)$ and, although to a minor degree also between INQoL index and Mental Health Index $(r=-0.5124$; $P<0.0001)$.

Added value of INQoL versus SF-36 When considering comparable domains of INQoL and SF-36 (pain versus bodily pain; weakness versus vitality; fatigue versus vitality; activity versus physical functioning; and emotion versus mental health) with respect to muscle strength assessment (MMRC) regression analysis showed a somewhat stronger correlation using INQoL rather than SF-36 scores for the following domains: Pain, Weakness, and Fatigue and a worse association for the domains Activity and Emotion (Table 8).

\section{Discussion}

We have translated in Italian and adapted the original UK version of INQoL and have tested it in a large and well-characterized sample of patients. We have demonstrated that this questionnaire is reliable, valid, and practical to assess QoL in adult patients with muscle diseases, thus confirming and extending data obtained in the United Kingdom in a smaller sample. 
Table 7 Correlations between INQoL - SF36 domains

\begin{tabular}{lllr}
\hline INQoL & SF-36 & $\begin{array}{l}\text { Correlation } \\
\text { coefficient }\end{array}$ & $P$-value \\
\hline Pain & Bodily pain & -0.7942 & $<0.0001$ \\
Pain & Role physical & -0.4957 & $<0.0001$ \\
Pain & Physical functioning & -0.4105 & $<0.0001$ \\
Weakness & Role physical & -0.4778 & $<0.0001$ \\
Weakness & Physical functioning & -0.7238 & $<0.0001$ \\
Weakness & Vitality & -0.5371 & $<0.0001$ \\
Fatigue & Role physical & -0.5959 & $<0.0001$ \\
Fatigue & Physical functioning & -0.6678 & $<0.0001$ \\
Fatigue & Vitality & -0.6228 & $<0.0001$ \\
Locking & Role physical & -0.2214 & 0.0026 \\
Locking & Physical functioning & -0.2017 & 0.0062 \\
Activity & Physical functioning & -0.7117 & $<0.0001$ \\
Activity & Social functioning & -0.4741 & $<0.0001$ \\
Relation & Social functioning & -0.4507 & $<0.0001$ \\
Emotion & Role emotional & -0.4949 & $<0.0001$ \\
Emotion & Mental health scale & -0.5124 & $<0.0001$ \\
\hline
\end{tabular}

Table 8 Correlations between selected scales from INQoL and SF36 with MMRC

\begin{tabular}{lrllr}
\hline INQoL & & & \multicolumn{2}{l}{ SF-36 } \\
\cline { 1 - 1 } Domain & \multicolumn{1}{c}{$R$} & & Domain & \multicolumn{1}{l}{$R$} \\
\hline Pain & 0.14499 & & Bodily pain & -0.08303 \\
Weakness & -0.21152 & & Vitality & 0.02014 \\
Fatigue & -0.22645 & & Physical functioning & 0.33986 \\
Activity & -0.12128 & & Mental health score & 0.14156 \\
Emotion & -0.00926 & & \\
\hline
\end{tabular}

Considering the domains/scales of the questionnaire determining INQoL index (Activities, Independence, Relationships, Emotions, and Body image), we confirmed that Activity and Independence seem to affect QoL more than the others.

When stratified by diseases, QoL perception appears to be better in group A (the myotonic dystrophies) compared to the other muscle disease groups considered in the study. It is well known that patients with myotonic dystrophy have a dysexecutive frontal syndrome [24-30], reduced initiative, difficulties with planning strategies, and apathy, and we may speculate that this in part justifies the patient's better perception of QoL than what would be expected by the objective and functional limitations present compared to other similarly affected patients.

As expected from our clinical experience and the UK data [10], disease duration is the parameter that most affects the patient's perception of QoL. In agrement with previous data, women have a worse QoL perception than men $[19,20]$. In contrast to literature data in other disorders, age [21,24] and education [31] do not seem to have an impact on the patient's perception of QoL $[22,23]$.
To determine whether INQoL assessed in a valid and reliable way physical and mental health concepts we tested the association with SF36 a well-known standardized HR-QoL generic questionnaire. We first tested the direction and strength of association between INQoL and the two summary scores of the SF36 indices. We then assessed the association between relevant domains of the two questionnaires (eg, the pain scales); eventually, we also tested the comparative capability of the two questionnaires in terms of strength of correlations between relevant scales and an objective measure of physical strength.

When considering the summary indices, results suggest that INQoL better captures physical than mental health concepts. When considering relevant homologous domains, all correlations were in the expected negative direction and most showed a substantial correlation, although again physical scales more strongly correlated than mental ones. The domain 'Locking', in INQoL, does not correlate with any of the physical domains of SF-36. This is a very specific muscle symptom confined to a restricted number of patients with muscle diseases (only group A in our study population) which cannot find a comparable health concept/scale in SF-36. Of note is the low correlation between INQOL relationships and the SF-36 social functioning scales. A possible explanation may be attributed to the fact that SF36 social functioning is known to be the less performing domain of the SF-36. The better association observed with the Physical Health Index of SF-36 compared to the Mental Health Index again emphasizes that INQoL is more sensitive in capturing the physical limitations because of the muscle condition.

Despite the observations outlined earlier, there are also several limitations of this study that need to be considered.

The first one regards INQoL construct. The structure of INQoL was derived from the ICIDH-2 model of disease which incorporates the concepts of impairment, activity, and participation. In this respect, a concern may be that INQoL reflects a measure of physical disability rather than of quality of life. However, the scores related to symptom severity are separated in the INQoL structure from the scores related to the impact on disease which constitutes quality of life. The score from each domain is actually weighted by the perceived importance assigned by the participant, and the final summary score from each section is presented as a percentage of the maximum detrimental impact.

Another concern may regard the heterogeneity of the sample considered. Our study population includes a variety of different muscle disorders that, although 
heterogeneous, share the property of physical disability, usually attributed to progressive muscle weakness.

Criterion validation only occurred in the patients with myotonic dystrophy. Patients with myotonic dystrophy may present only distal muscle weakness or both proximal and distal muscle weakness as disease progresses. We cannot definitely conclude that these results apply to other forms of myopathy, but the results are encouraging and obtained on a large sample size $(n=422)$. However, further testing in other disease groups is necessary.

Another limitation of our study regards the fact we have not unfortunately yet had the opportunity to study responsiveness of INQoL. This is a fundamental attribute to assess this QoL instrument to detect change over time and will be our next step.

In conclusion, the muscle disease specificity of INQoL, the documented content, and construct validity in the UK population, together with the acceptable expected burden for patients prompted us to translate the questionnaire and proceed for validation in Italy. Our results have documented good reliability and internal consistency, satisfactory psychometric and known-group validity in Italian adult patients with muscle diseases. Concurrent validity with SF-36 indicates that INQoL is more sensitive in capturing physical rather than psychological health concepts, and some of its scale are more strongly associated with an objective and standardized measure of muscle strength when compared with the relevant scales from the SF-36.

\section{Acknowledgements}

This work was supported by a UILDM-Telethon grant given to V. Sansone (GUP05001).

\section{Supporting Information}

Additional Supporting Information may be found in the online version of this article:

Table S1. Summary of diagnostic criteria for the disease groups considered and number of patients included in the study for each muscle disease.

Table S2. Sections of INQoL questionnaire.

Please note: Wiley-Blackwell are not responsible for the content or functionality of any supporting materials supplied by the authors. Any queries (other than missing material) should be directed to the corresponding author for the article.

\section{References}

1. Ware JE Jr, Gandek B, Kosinski M, et al. The equivalence of SF-36 summary health scores estimated using standard and country-specific algorithms in 10 countries: results from the IQOLA Project. InternationalQuality of Life Assessment. J Clin Epidemiol 1998; 51: 1167-1170.

2. Ware JE Jr, Kosinski M, Gandek B, et al. The factor structure of the SF-36 Health Survey in 10 countries: results from the IQOLA Project. International Quality of Life Assessment. J Clin Epidemiol 1998; 51: 1159-1165.

3. Keller SD, Ware JE Jr, Bentler PM, et al. Use of structural equation modeling to test the construct validity of the SF-36 Health Survey in ten countries: results from the IQOLA Project. International Quality of Life Assessment. J Clin Epidemiol 1998; 51: 1179-1188.

4. Apolone G, Mosconi P. The Itlian Sf-36 Health Survey: translation, validation and norming. J Clin Epidemiol 1998; 51: 1025-1036.

5. Boyer F, Morrone I, Laffont I, et al. Health related quality of life in people with hereditary neuromuscular diseases: an investigation of test-retest agreement with comparison between two generic questionnaires, the Nottingham healt profile and the short form-36 item. Neuromuscul Disord 2006; 16: 99-106.

6. Dallmeijer AJ, Dekker J, Knol DL, et al. Dimensional structure of the SF-36 in neurological patients. J Clin Epidemiol 2006; 59: 541-543.

7. Ahlstrom G, Gunnarsson LG, Kihlgren A, et al. Respiratory function, electrocardiography and quality of life in individuals with muscular dystrophy. Chest 1994; 106: 173-179.

8. Ahlstrom G, Gunnarsson LG. Disability and quality of life in individuals with muscular dystrophy. Scand $J$ Rehabil Med 1996; 28: 147-157.

9. Coons SJ, Rao S, Keininger DL, et al. A comparative review of generic quality-of-life instruments. Pharmacoeconomics 2000; 17: 13-35.

10. Vincent KA, Carr AJ, Walburn J, et al. Construction and validation of a quality of life questionnaire for neuromuscular disease (INQoL). Neurology 2007; 68: 10511057.

11. Folstein MF, Folstein SE, McHugh PR. "Mini-mental state". A practical method for grading the cognitive state of patients for the clinician. J Psychiatr Res 1975; 12: 189198.

12. Medical Research Council of the United Kingdom. Aids to Examination of the Peripheral Nervous System Memorandum No 45. Palo Alto, CA: Pedragon House, 1978.

13. Kilmer DD, Abresch RT, Fowler WM Jr. Serial manual muscle testing in Duchenne muscular dystrophy. Arch Phys Med Rehabil 1993; 74: 1168-1171.

14. Scientific Advisory Committee of the Medical Outcomes Trust. Assessing health status andquality-of-lfe instruments: attributes and review criteria. Qual Life Res 2002; 11: 193-205.

15. Lohr KN, Aaronson NK, Alonso J, et al. Evaluating quality-of-life and health status instruments: development of scientific review criteria. Clin Ther 1996; 18: 979-991.

16. Shrout PE, Fleiss JL. Intraclass correlations: uses in assessing rater reliability. Psychol Bull 1979; 86: 420-428.

17. Bland JM, Altman DG. Statistical methods for assessing agreement between two methods of clinical measurement. Lancet 1986; i: $307-310$.

18. Cronbach L. Coefficient alpha and the internal structure of tests. Psychometrika 1951; 16: 297-334.

19. Casellas F, Rodrigo L, Vivancos JL, et al. Factors that impact health-related quality of life in adults with celiac 
disease: A multicenter study. World J Gastroenterol 2008; 14: $46-52$

20. Vázquez I, Valderrábano F, Fort J, et al. Psychosocial factors and health-related quality of life in hemodialysis patients. Qual Life Res 2005; 14: 179-190.

21. Jakobsson U. Using the 12-item Short Form health survey (SF-12) to measure quality of life among older people. Aging Clin Exp Res 2007; 19: 457-464.

22. Strombeck B, Ekdahl C, Manthorpe R, et al. Healthrelated quality of life in primary Sjogren's syndrome, rheumatoid arthritis and fibromyalgia compared to normal population data using SF-36. Scand $J$ Rheumatol 2000; 29: 20-28.

23. Haywood KL, Garratt AM, Fitzpatrick R. Quality of life in older people: a structured review of generic self-assessed health instruments. Qual Life Res 2005; 14: 1651-1668.

24. Harper PS. Myotonic dystrophy: Major Problems in Neurology, 3rd edn. London: WB Saunders Co, 2001.

25. Meola G, Sansone V, Perani D, et al. Reduced cerebral blood flow and impaired visual-spatial function in proximal myotonic myopathy. Neurology 1999; 53: 1042-1050.
26. Meola G, Sansone V, Perani D, et al. Executive dysfunction and avoidant personality trait in myotonic dystrophy type 1 (DM1) and in proximal myotonic myopathy (PROMM/DM2). Neuromuscul Disord 2003; 13: $813-821$.

27. Sansone V, Gandossini S, Cotelli M, et al. Cognitive impairment in adult myotonic dystrophies: a longitudinal study. Neurol Sci 2007; 28: 9-15.

28. Meola G, Sansone V. Cerebral involvement in myotonic dystrophies. Muscle Nerve 2007; 36: 294-306.

29. Modoni A, Silvestri G, Pomponi MG, et al. Characterization of the pattern of cognitive impairment in myotonic dystrophy type 1. Arch Neurol 2004; 61: 1943-1947.

30. Antonini G, Soscia F, Giubilei F, et al. Health-related quality of life in myotonic dystrophy type 1 and its relationship with cognitive and emotional functioning. J Rehabil Med 2006; 38: 181-185.

31. Lopes AA, Bragg-Gresham JL, Goodkin DA, et al. Factors associated with health-related quality of life among hemodialysis patients in the DOPPS. Qual Life Res 2007; 16: $545-557$. 